\title{
Content Analysis of Tweets of Pregnant Women with Diabetes
}

\author{
Iris Thiele Isip-Tan, ${ }^{1}$ Helen V. Madamba ${ }^{2}$ and Rene James P. Balandra Jr. ${ }^{3}$ \\ ${ }^{1}$ Department of Medicine, College of Medicine and Philippine General Hospital, University of the Philippines Manila \\ ${ }^{2}$ College of Medicine, Cebu Doctors University \\ ${ }^{3}$ National Telehealth Center, National Institutes of Health, University of the Philippines Manila
}

\begin{abstract}
Objectives: Describe health beliefs of pregnant women with diabetes using tweets. Describe how information on diabetes in pregnancy is shared on Twitter.

Methods: Tweets by women with diabetes were identified from Symplur Signals. "Status" tweets were mapped to the Health Belief Model. Tweets by women with preexisting diabetes and gestational diabetes (GD) were analyzed separately. Links within tweets were surveyed for the Health on the Net (HON) Foundation seal.
\end{abstract}

Results: Women with GD tweeted about cravings and the connection of high carbohydrate meals with big babies. Perceived barriers included food restriction, hunger, lab tests, clinic consults and blood glucose monitoring. Perceived benefits of blood glucose testing and a healthy diet were linked to healthy babies. Blood glucose monitoring, weight gain, and age of gestation were cues to action. Perceived barriers of women with preexisting diabetes were feelings of helplessness, loss of control, and anger. Nine domains (9.7\%) had the HON Code seal. Women with preexisting diabetes shared blog posts. Women with GD shared links from organizations.

Conclusion: Women with GD and preexisting diabetes had differing perceptions of susceptibility, severity, barriers, benefits, cues to action, and self-efficacy; and shared links to information differently on Twitter.

Key Words: gestational diabetes, pregestational diabetes, Twitter, health belief model

\section{Introduction}

Pregnancy can be difficult enough without preexisting diabetes or the added burden of a diagnosis of gestational

Presented as a poster at 18th ASEAN Federation of Endocrine Societies Congress, Dec. 11-13, 2015, Kuala Lumpur Convention Centre, Kuala Lumpur, Malaysia.

Finalist at the 2015 Stanford Medicine X \& Symplur Signal Research Challenge. Announced Aug. 17, 2015. http://medicinex.stanford.edu/2015/08/17/stanfordmedicine-x-symplur-signals-research-challenge-winner-announcement/

Corresponding author: Iris Thiele Isip-Tan MD, MSc

Department of Medicine

Philippine General Hospital

University of the Philippines Manila

Taft Ave., Ermita, Manila 1000 Philippines

Telephone: +632 3105349

Email: icisiptan@up.edu.ph diabetes mellitus. Women with GD are time-pressured to adjust their dietary habits and lifestyle to maintain good glycemic control. Few studies have examined how these women handle the diagnosis.

A systematic review on determinants and barriers for GD services from screening to postpartum follow-up by Nielsen ${ }^{1}$ et al. showed that while women are motivated to protect their baby, there are many challenges and "precious little is known about health system or societal factors that hinder compliance and what can be done to improve it."

A focus group discussion among Vietnamese women with gestational diabetes ${ }^{2}$ explored six themes: diagnosis and ethology of GD, effects of GD on pregnancy, dietary changes, blood glucose monitoring, breastfeeding, and sources of information. The authors found that women were confused about the diagnosis, its effects on the baby and dietary instruction. Carolina ${ }^{3}$ et al. identified barriers that complicated self-management of GD: time pressures, physical and social constraints, limited comprehension of requirements, and insulin as an easier option.

Women with GD have also said that physical limitations and restrictions, lack of resources, energy and time were barriers to exercise during pregnancy; while social support, access to resources, information, proper diet, scheduling, and weather were facilitators. ${ }^{4}$

Twitter is a social network that lets users send and read "tweets," which are short messages of 140 characters. In 2015, Twitter had an average of 320 million monthly active users globally. ${ }^{5}$ Various health issues have been studied on Twitter by analyzing tweets about alcohol use, ${ }^{6}$ sleep problems, $^{7}$ smoking cessation, ${ }^{8}$ suicide, ${ }^{9}$ breast cancer, ${ }^{10}$ allergic rhinoconjunctivitis, ${ }^{11}$ and obesity. ${ }^{12}$

The previously cited studies on women with GD used structured interviews or focus group discussions which limited the sample size. Twitter provides access to tweets of pregnant women with diabetes. These tweets may provide clues as to their information needs and health behavior.

\section{Objectives}

1. Describe health beliefs of pregnant women with diabetes using tweets.

2. Describe how information on diabetes in pregnancy is shared on Twitter. 


\section{Methods}

Table 1 shows the search strategies used to generate tweet transcript reports from Symplur Signals from 1 Jan 2010 12:00 AM to 1 Jan 2015 12:00 am Asia/Shanghai time. Tweets were filtered to identify tweets by patients. However, many Twitter profiles in the database were not tagged as by patients. Hence, tweet transcripts from strategies 3 and 7 were manually searched to identify tweets by pregnant women with diabetes. Duplicate tweets were excluded. Tweets were excluded if the Twitter profile data identified a male, healthcare professional or organization.

A single investigator coded the tweets using Dann's Twitter content classification. ${ }^{13}$ Status tweets answer the question "What are you doing now?" which Dann further divides into the following:

a. Personal - positive or negative sentiment in the form of personal opinion or emotional status

b. Temporal - content referencing specific dates, times, statements of temporal nature (waiting) and temporal action ("Time to")

c. Location - geographic references and location statements, including statements of traveling, location change

d. Mechanical - statements relating to any form of technology or mechanical systems (cars, phones, printers and photocopiers)

e. Physical - sensory experiences of a physical nature

f. Work - reference to work related activity

g. Automated - status announcements triggered by third party applications such as media players, games or software

h. Activity - activity statements answering "What are you doing now?"

Two investigators independently mapped each "Status" tweet to a construct of the Health Belief Model ${ }^{14}$ : perceived susceptibility, perceived benefits, cues to action, perceived barriers, perceived severity, and self-efficacy. Differences were resolved by consensus. Tweets indexed by hashtags "pregnancy" and "diabetes" were likely posted by women with pregestational diabetes and were analyzed separately from tweets labeled by the hashtag "gestational diabetes" likely posted by women who had been diagnosed with diabetes during the pregnancy, as their health behavior was plausibly different.

"Pass along" tweets endorse content and are further divided into:

a. RT - any statement reproducing another Twitter status using the via@ or RT protocol

b. UGC - links to content created by the user (blog/ video/picture)

c. Endorsement - links to web content not created by the sender

Links within tweets classified as user-generated content (UGC) and endorsement were included for analysis.
Retweets (RT) were excluded. Unique domains of these links were surveyed for the Health on the Net $(\mathrm{HON})$ Foundation $^{15}$ seal, which ensures quality, objectivity, and transparency of medical information online.

\section{Results}

The combined search strategies yielded 6,433 tweets. Table 1 shows how many tweets each search strategy yielded. A total of 1,024 tweets were identified as posted by patients. The investigators scanned 4,260 tweets to manually identify tweets by pregnant women with diabetes, which yielded an additional 524 tweets. Thus 1,548 tweets were coded for content.

Seventy-nine women with pregestational diabetes contributed a total of 184 status tweets. Twenty-four of these women explicitly stated on their Twitter profiles that they had type 1 diabetes. Sixteen women with type 1 diabetes also wrote blogs. Location was not stated in 27 Twitter profiles. Majority of women who included location on Twitter profiles were from the United States.

Two hundred and six women contributed 292 status tweets on GD. Of these, 12 had Twitter handles that contained variations of mom or mama, 24 had profile pictures with children and 77 explicitly mentioned that they were mothers on their profiles. Location data was available for only 119 profiles. Most women were in the United States but there was one woman each from Singapore and Japan and four women from the Philippines.

Table 2 shows the number of status tweets classified by content and mapped to constructs of the Health Belief Model. The distribution of tweets according to constructs differed somewhat between women with GD and those with pregestational diabetes (Figure 1). While tweets relating to barriers predominated in both groups, a greater percentage of such tweets were seen for women with GD. Tweets on perceived severity and cues to action were fewer for women with GD.

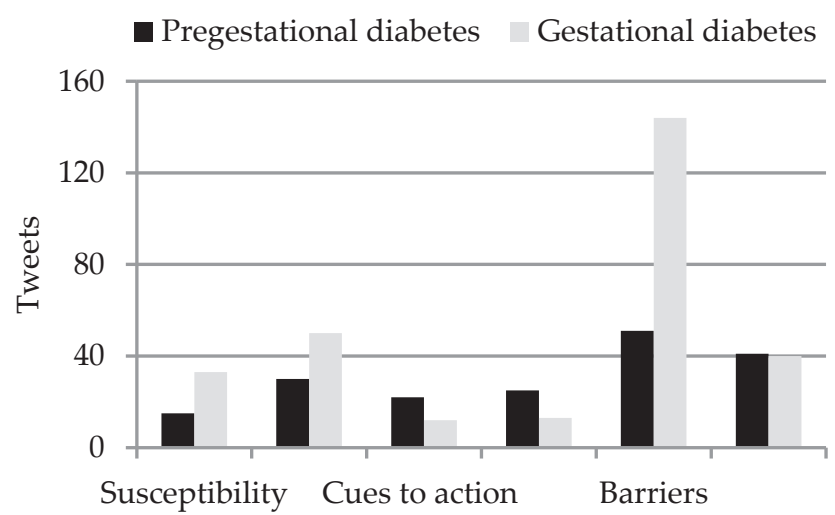

Figure 1. Distribution of status tweets mapped to constructs of the Health Belief Model comparing women with GD and pregestational diabetes. 
Table 1. Classification of tweets included for mapping to the Health Belief Model

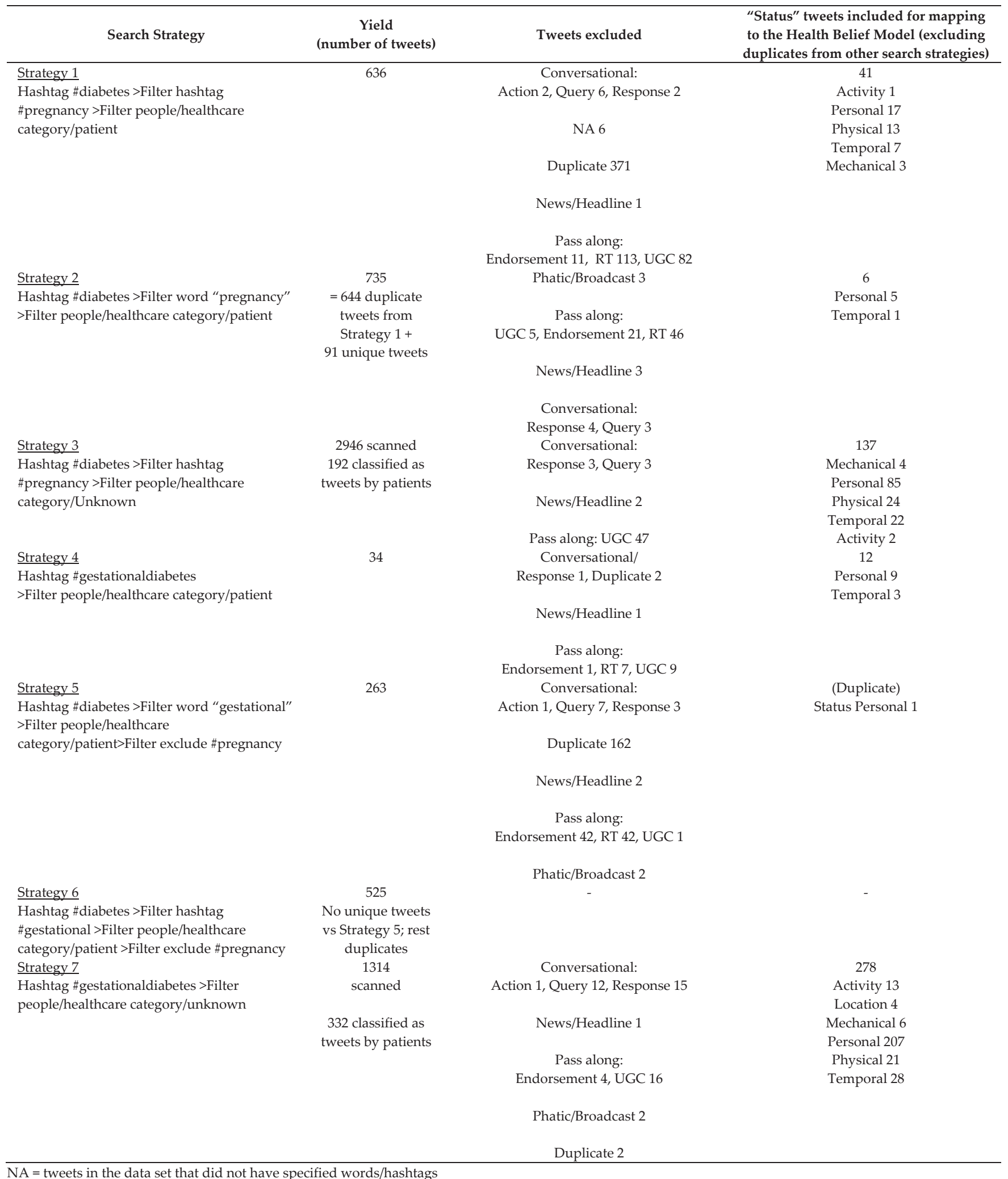


Table 2. Summary of status tweets classified by content and as mapped to constructs of the Health Belief Model

\begin{tabular}{|c|c|c|}
\hline \multicolumn{3}{|c|}{ Pregestational diabetes $\mathrm{n}=184$} \\
\hline Perceived susceptibility & Perceived benefits & Cues to action \\
\hline Belief about the chances of experiencing a risk or & Belief in efficacy of the advised action to reduce & Strategies to activate "readiness" \\
\hline getting a condition or disease & risk or seriousness of impact & $\mathrm{n}=22(11.9 \%)$ \\
\hline $\mathrm{n}=15(8.1 \%)$ & $\mathrm{n}=30(16.3 \%)$ & Mechanical $n=4$ \\
\hline Personal $\mathrm{n}=10$ & Personal $n=20$ & Personal n=2 \\
\hline Physical n=2 & Temporal $n=5$ & Physical n=6 \\
\hline Temporal $\mathrm{n}=3$ & Physical n=5 & Temporal $\mathrm{n}=10$ \\
\hline Perceived severity & Perceived barriers & Self-efficacy \\
\hline Belief about how serious a condition and its & Belief about tangible and psychological costs of & Confidence in one's ability to take action \\
\hline sequelae are & the advised action & $\mathrm{n}=41(22.3 \%)$ \\
\hline $\mathrm{n}=25(13.6 \%)$ & $\mathrm{n}=51(27.7 \%)$ & Activity $n=3$ \\
\hline Personal n=14 & Mechanical n=2 & Mechanical n=1 \\
\hline Physical n=8 & Personal $n=34$ & Personal n=27 \\
\hline Temporal $\mathrm{n}=3$ & Physical n=12 & Physical n=4 \\
\hline & Temporal n=3 & Temporal $\mathrm{n}=6$ \\
\hline \multicolumn{3}{|c|}{ Gestational diabetes $n=292$} \\
\hline Perceived susceptibility & Perceived benefits & Cues to action \\
\hline $\mathrm{n}=33(11.3 \%)$ & $\mathrm{n}=50(17.1 \%)$ & $\mathrm{n}=12(4.1 \%)$ \\
\hline Personal $n=28$ & Activity $n=1$ & Mechanical n=2 \\
\hline Activity $n=2$ & Mechanical $n=1$ & Personal n=5 \\
\hline Location $n=1$ & Personal $n=40$ & Temporal $n=5$ \\
\hline Mechanical n=1 & Physical n=1 & \\
\hline Temporal n=1 & Temporal $n=7$ & \\
\hline Perceived severity & Perceived barriers & Self-efficacy \\
\hline $\mathrm{n}=13(4.4 \%)$ & $\mathrm{n}=144(49.3 \%)$ & $\mathrm{n}=40(13.7 \%)$ \\
\hline Personal n=11 & Activity $n=7$ & Temporal $n=5$ \\
\hline Physical n=1 & Temporal $n=12$ & Personal $n=28$ \\
\hline \multirow[t]{4}{*}{ Temporal n=1 } & Location $n=3$ & Activity $n=3$ \\
\hline & Mechanical n=2 & Physical n=4 \\
\hline & Personal n=104 & \\
\hline & Physical n=16 & \\
\hline
\end{tabular}

Perceived susceptibility is the belief about the chances of experiencing a risk or getting a condition or disease. ${ }^{14}$ Women with GD expressed shock and dismay on diagnosis.

Logically I know \#GestationalDiabetes is not my fault...pregnancy hormones make it impossible to be logical \#PregnancyProblems \#hormonal

I have gestational diabetes. I am in shock. Advice? \#gestationaldiabetes \#2ndbaby \#shouldibefreakingout

Failed the 3 hour test....so I have gestational diabetes AGAIN... Yeah monthly visits to EVMS \#gestationaldiabetes

Guess who has Gestational Diabetes... :-( \#pregnancy \#twinpregnancy \#twins \#pregnantwithtwins \#gestationaldiabetes

Why do I have cravings for sweets a week before my Glucose Tolerance test?! :P Super worried about \#gestationaldiabetes :/ Help me, Oh Lord!

Damn Pregnancy hormones! Kung kelan malapit na! T_T \#gestationaldiabetes \#30weeks

Perceived severity is the belief about how serious a condition and its sequelae are. ${ }^{14}$ This was conveyed by tweets about cravings and the connection of high carbohydrate meals with big babies.

So, my baby weighs 9 pounds at only 36 weeks gestation. Hmmmmm..... \#pregnancy \#Slightly Apprehensive \#birth \#gestationaldiabetes
Mom has had way way way too many carbs today. Poor baby is gonna be on sugar overload. \#Gestational Diabetes :(

Heading to an ultrasound to see how big baby girl is... eeeeeek! \#29weeks \#gestationaldiabetes

Perceived barriers refer to belief about tangible and psychological costs of the advised action. ${ }^{14}$ Tweets included were about food restriction and hunger $(61 \%)$, dealing with emotions $(9.0 \%)$, blood glucose monitoring $(7.6 \%)$, clinic consults $(6.2 \%)$, and lab tests (4\%).

Just did the worlds most boring grocery shop because I have \#gestationaldiabetes Fun times..!

$I$ just ate a sugar free $P B \in \mathcal{E} J$. \#disgusting \#sugarfreejelly \#gestationaldiabetes

What $i$ would do for a $g$ damn brownie right now \#gestationaldiabetes \#pregnantladyrage

Cheating on a diet and feeling like a murderer while you frantically hide the evidence! \#gestationaldiabetes \#sucks \#nosugar \#thestruggle

Omg today is national cream filled donut day and I can't participate because of stupid \#gestationalDiabetes, that's just crap!

I am sick of this frickin' diet... Just hate it \#PregnancyProblems \#gestationaldiabetes \#GDM \#pregnant 
That low point when you find yourself crying because you can't eat what you want. \#Gestational Diabetes \#PregoProbs \#Fat

Feel like vomiting after the horrible glucose drink for the \#gestationaldiabetes test. I got pressured into it! \#industry \#medicine

Why did I schedule my glucose test for the morning after Halloween?? \#gestationaldiabetes

I just had to poke 5 different fingers to get a reading. I wish there was an easier way. \#ow \#gestationaldiabetes

I get to prick my finger like Sleeping Beauty, except I have to do it $4 x$ a day Eamp; count carbs for six weeks. \#GestationalDiabetes What fun.

First day testing blood sugars... This sucks \#gestationaldiabetes \#nomoreoreos

Sometimes pricking your finger to test your blood REALLY freaking hurts. \#gestationaldiabetes \#ouch

I don't trust the newbie sonographers measurements or over cautious junior docs judgement. Going for 2 nd opinion. \#GestationalDiabetes

In the doctors having my GTT for gestational diabetes, only another hour to go! \#bored \#GTT \#GestationalDiabetes \#preggoproblems

Spending too long waiting in doctors offices \#thirdtrimester \#pregnancy \#gestationaldiabetes

Next person who tells me \#gestationaldiabetes can be controlled with diet and exercise alone will die. That's not true at all. \#SOBs

This informational \#gestationaldiabetes video actually insults my intelligence. Cakes and cookies are high in sugar? You don't say!

Watching a video on \#gestationaldiabetes that clearly was made for morons who live under rocks.

Perceived benefits refer to the belief in the efficacy of the advised action to reduce risk or seriousness of impact. ${ }^{14}$ This was expressed in tweets on blood glucose testing and following a healthy diet that were linked to healthy babies.

Meat sauce over baked garlic cabbage. \#lowcarb \#gestationaldiabetes

Healthy snack. \#29weekspregnant \#gestational diabetes \#healthy \#snack \#cucumber \#carrot \#yoghurt

$I$ will do anything for love -- poke my finger $7 x$ a day and get shots $4 x$ a day \#gestationaldiabetes \#babylove

Found out I can eat air popped popcorn!! \#gestationaldiabetes woohoo! (Little things make me happy these days)

Blood glucose monitoring, weight gain, and age of gestation were cues to action.

Steadily gaining a pound a week for the last 6 . Thank God that slowed down. \#gestationaldiabetes isn't all bad. \#33weeks \#pregnant
Stupid elevated after lunch sugar number. Hitting the treadmill. \#gestationaldiabetes \#32weeks

\#30weekspregnant \#babybump \#gestationaldiabetes \#walking \#stayfit \#stayhealthy \#iloveexercise

Some of the women proclaimed confidence in managing their condition (self-efficacy), and shared their success on Twitter.

Multigrain cheerios with soy milk for breakfast; carrots and ranch for a snack. \#Lifestylechanges \#gestationaldiabetes

A \#gestationaldiabetes breakthrough. Homemade rye flour fruit scones, low fat double cream $\mathcal{E}$ fresh strawberries!

Hopped on the treadmill for 30 min after work, made dinner, AND walked the dogs tonight. Woooooot! \#kickass \#gestationaldiabetes \#30weeks

Been monitoring my blood sugar today. Whooped that elevated sugar's butt! \#gestationaldiabetes \#aintgotshitonme \#pregnancyproblems

Tested my sugar after dinner and it was well within range. \#Victorious \#GestationalDiabetes \#CelebrateThe SmallThings

Proud that I've learned to inject insulin by myself have overcome my deep fear of needles! \#gestational diabetes

Two miles done and done. Before work. Before 6am. Woooo! \#gestationaldiabetes \#kickass \#pregnant

Tweets from women with preexisting diabetes differed somewhat from those with GD. Perceived barriers were more emotional (33.3\%) such as feelings of helplessness, loss of control and anger were more common than tweets about dietary restrictions (23.5\%).

High blood sugar and a pod alarm. Emergency pod change. Ugh. Not how I'd like to be woken up. \#pregnancy \#diabetes

I miss my mum and sisters.feeling emotional $n$ scared. being pregnant without them to help is no fun! \#diabetes \#pregnancy

Well.. I could use a vacation from needles and notas-low-as-the-drs-would-like blood sugars today...* sigh* \#diabetes \#pregnancy \#insulin

Diabetes sucks! \#diabetes \#diabetics \#bloodsugars \#pregnancy \#diabeticpregnancy \#troubles

WHAT?! After 50 units of \#insulin, a $12.1 \mathrm{mmol} / \mathrm{L}$ result?!?! UGH!! \#pregnancy \#diabetes

How many hours have I wasted in the doctor's office/waiting room this week? It's a lot \#diabetes \#pregnancy \#thirdapptthisweek

I don't want to do this anymore. Anyone want to take over my \#diabetes or \#pregnancy? I'll let you pick. Wait, could I get 2 volunteers?

pregnancy insulin resistance is FUN! I miss carb loading :( \#T1D \#pregnancy \#diabetes 
Self-efficacy was evident in tweets declaring ability to adjust insulin as needed.

I think the insulin resistance is starting to kick in. Upping my basal to (hopefully) stay ahead of it. \#diabetes \#pregnancy

My two week BG average is 108-take that pregnancy insulin resistance!! \#diabetes \#pregnancy \#hardwork \#35weeks

Doctor's appt went great! Blood glucose a bit high, so calling my nurse and I'm sure upping my \#Insulin dose. \#diabetes \#pregnancy

The data set contained 239 links from 93 unique domains (Table 3). Only 9 domains (9.7\%) had the HON Code seal. Women with preexisting diabetes shared blog posts $(67 \%)$ in tweets classified under user-generated content, compared to women with GD who shared links from organizations or news sites in endorsement tweets $(64 \%)$.

\section{Discussion}

Pre-pregnancy care for women with preexisting diabetes is effective in reducing perinatal mortality and improving rates of congenital malformation, but low utilization of such programs remains a major obstacle. ${ }^{16}$ Knowing the health beliefs of these women may be helpful.

The majority of tweets of women with GD were about perceived barriers in diagnosis and lab testing, diet, and glucose monitoring. This may be because women with GD have limited time to handle their diagnosis and acquire skills for self-management. Women with preexisting diabetes tweeted more about emotional barriers, perhaps because of the chronicity of their condition.
Women with gestational diabetes tweet links from organizations or news sites, perhaps sharing what they have read as they try to understand their condition. In contrast, those with pregestational diabetes share their experiences through blog posts as pregnancy is a chapter in their chronic disease journey.

The Health on the Net (HON) Foundation has eight principles that govern the code of conduct for medical and health websites: authoritative, complementarity, privacy, attribution, justifiability, transparency, financial disclosure, and advertising policy. Websites that apply for the HON code seal adhere to these principles. While less than $10 \%$ of links shared via tweets in the data set have the HON code seal, this does not mean that the links shared are not adhering to accepted standards as application for HON code certification is voluntary. The HON code will also likely not be applicable to blogs that talk about the experience of having diabetes in pregnancy as these do not claim to be sources of medical information per se.

Twitter users are likely not representative of women with GD and pregestational diabetes. More women with type 1 diabetes were included in the sample of tweets of those with preexisting diabetes. Women with type 2 diabetes were not explicitly identified using Twitter profile data. Profiling the women whose tweets were included in the data set was limited by the paucity of information on their Twitter profiles. There were very few Asian women included in the data set. Nonetheless, this content analysis provides information on the health beliefs of pregnant women with diabetes.

Tweets as representations of healthcare conversations has previously been studied. ${ }^{17,18,19}$ This study and others like it adds the patients' voice to the biomedical literature.

Table 3. Links and unique domains included in the study

\begin{tabular}{|c|c|c|c|}
\hline Search Strategy & $\begin{array}{c}\text { Yield } \\
\text { (Links in "Pass along" tweets) }\end{array}$ & Unique Domains & HON-certified domains \\
\hline Strategy 1 & Endorsement 11 & 20 & UGC 2 \\
\hline Hashtag \#diabetes >Filter hashtag \#pregnancy & User generated content & Endorsement 9 & (https://diabetogenic.wordpress.com, \\
\hline$>$ Filter people/healthcare category/patient & (UGC) 82 & UGC 11 & textingmypancreas.com) \\
\hline limit 100 & & & $\begin{array}{c}\text { Endorsement } 1 \\
\text { (http://www.healthcentral.com/) }\end{array}$ \\
\hline Strategy 2 & Endorsement 21 & 21 & Endorsement 2 \\
\hline Hashtag \#diabetes >Filter word "pregnancy" & UGC 5 & Endorsement 19 & (http://www.tudiabetes.org/, \\
\hline >Filter people/healthcare category/patient & & UGC 2 & http://sixuntilme.com/) \\
\hline limit 100 & & & $\begin{array}{c}\text { UGC 1 } \\
\text { (http://www.ydmv.net/) }\end{array}$ \\
\hline$\underline{\text { Strategy } 3}$ & UGC 47 & 12 & 0 \\
\hline $\begin{array}{l}\text { Hashtag \#diabetes >Filter hashtag \#pregnancy } \\
>\text { Filter people/healthcare category/Unknown } \\
\text { limit } 300\end{array}$ & & UGC 12 & \\
\hline$\underline{\text { Strategy } 4}$ & Endorsement 1 & 2 & 0 \\
\hline Hashtag \#gestationaldiabetes & UGC 9 & Endorsement 1 & \\
\hline$>$ Filter people/healthcare category/patient & & UGC 1 & \\
\hline Strategy 5 & Endorsement 42 & 23 & Endorsement 2 \\
\hline $\begin{array}{l}\text { Hashtag \#diabetes > Filter word "gestational" } \\
>\text { Filter people/healthcare category/patient }\end{array}$ & UGC 1 & Endorsement 23 & $\begin{array}{l}\text { (http://www.emaxhealth.com/, } \\
\underline{\text { http://www.webmd.com) }}\end{array}$ \\
\hline Strategy 7 & Endorsement 4 & 15 & Endorsement 1 \\
\hline $\begin{array}{l}\text { Hashtag \#gestationaldiabetes } \\
>\text { Filter people/healthcare category/unknown }\end{array}$ & UGC 16 & $\begin{array}{l}\text { Endorsement 4, } \\
\text { UGC } 11\end{array}$ & (http://www.medicalnewstoday.com/) \\
\hline
\end{tabular}




\section{Conclusion}

Contact between physicians and pregnant women with diabetes are often limited during the pregnancy. These women may not express their feelings or difficulties during clinic consults. Examining tweets of pregnant women with diabetes has identified issues that are important to them. These include dietary restrictions, laboratory testing, glucose monitoring, and emotional distress. Content analysis of tweets showed that women with GD and preexisting diabetes had differing perceptions of susceptibility, severity, barriers, benefits, cues to action, and self-efficacy. Physicians following up these women can gain insight from this study as to what can be discussed more often during consultations and be able to provide more support.

Women with GD and those with preexisting diabetes shared links to information differently. As women with preexisting diabetes tweeted links to their own blog posts about their condition, the content of these blogs can be further investigated. Further studies can also look into which links from organisations or news sites tweeted by women with GD are more likely to be retweeted and shared. The findings from this study can also be useful in planning an information campaign for diabetes in pregnancy on social media.

\section{References}

1. Nielsen KK, Kapur A, Damm P, de Courten M, Bygbjerg IC. From screening to postpartum follow-up - the determinants and barriers for gestational diabetes mellitus (GDM) services, a systematic review. BMC Pregnancy Childbirth. 2014; 14:41.

2. Hirst JE, Tran TS, Do MA, Rowena F, Morris JM, Jeffery HE. Women with gestational diabetes in Vietnam: a qualitative study to determine attitudes and health behaviors. BMC Pregnancy Childbirth. 2012; 12:81.

3. Carolan M, Gill GK, Steele C. Women's experiences of factors that facilitate or inhibit gestational diabetes self-management. BMC Pregnancy Childbirth. 2012; 12:99.

4. Marquez DX, Bustamante EE, Bock BC, Markenson G, Tovar A, ChasenTaber L. Perspectives of Latina and non-Latina white women on barriers and facilitators to exercise in pregnancy. Women Health. 2009; 49(6):505-21.
5. Desilver D. 5 facts about Twitter at age 10 [Online]. 2016 [cited 2016 May]. Available from http://www.pewresearch.org/fact-tank/2016/03/18/5-factsabout- twitter-at-age-10/.

6. Cavazos-Rehg PA, Krauss MJ, Sowles SJ, Bierut LJ. “Hey Everyone, I'm Drunk." An Evaluation of Drinking-related Twitter Chatter. J Stud Alcohol Drugs. 2015; 76(4):635-43.

7. McIver DJ, Hawkins JB, Chunara R, et al. Characterizing sleep issues using Twitter. J Med Internet Res. 2015; 17(6):e140.

8. Chung JE. A smoking cessation campaign on Twitter: Understanding the use of Twitter and identifying major players in a health campaign. J Health Commun. 2016; 21(5):517-26.

9. Colombo GB, Burnap P, Hodorog A, Scourfield J. Analysing the connectivity and communication of suicidal users on Twitter. Comput Commun. 2016; 73(Pt B):291-300.

10. Kim E, Hou J, Han JY, Himelboim I. Predicting retweeting behavior on breast cancer social networks: network and content characteristics. J Health Commun. 2016; 21(4):479-86.

11. Gesualdo F, Stilo G, D'Ambrosio A, et al. Can Twitter Be a Source of Information on Allergy? Correlation of Pollen Counts with Tweets Reporting Symptoms of Allergic Rhinoconjunctivitis and Names of Antihistamine Drugs. PLoS One. 2015; 10(7):e0133706.

12. So J, Prestin A, Lee L, Wang Y, Yen J, Chou WYS. What do people like to "share" about obesity? A content analysis of frequent retweets about obesity on Twitter. Health Commun. 2016; 31(2):193-206.

13. Dann S. Twitter content classification. First Monday [Online]. 2010. [cited 2015 June]. Available from http://journals.uic.edu/ojs/index.php/fm/ article/view/2745

14. Champion VL, Skinner SG. The Health Belief Model. In: Glanz K, Rimer BK, Viswanath K (eds). Health Behavior and Health Education: Theory, Research and Practice, 4th ed. San Francisco: John Wiley \& Sons; 2008. pp. 45-66.

15. Health on the Net Foundation Code [Online]. [cited 2015 Mar]. Available from http://www.hon.ch/HONcode/Patients/Visitor/visitor.html.

16. Wahabi HA, Alzeidan RA, Esmail SA. Pre-pregnancy care for women with pre-gestational diabetes mellitus: a systematic review and metaanalysis. BMC Public Health. 2012; 12:792.

17. Xu WW, Chiu I, Chen Y, Mukherjee T. Twitter hashtags for health: applying network and content analyses to understand the health knowledge sharing in a Twitter-based community of practice. Quality \& Quantity. 2015; 49(4):1361-80.

18. Katz M, Utengen A, Anderson PF, et al. Disease-specific hashtags for online communication about cancer care. JAMA Oncol. 2016; 2(3):392-4.

19. Harmel M, Young K. e-patients in Twitter hashtag communities. J Participat Med. 2013; 5:e22. 